\title{
6. Zusammenfassende Schlussbetrachtung
}

Die beginnende Industrialisierung des Großraums Paris entlang der Seine und die starke französische Binnenmigration hatten den ländlichen Raum vor den Stadttoren von Paris bis zum Ersten Weltkrieg nachhaltig verändert. Anstelle von kleinen landwirtschaftlichen Betrieben und freien Flächen entstanden nun Manufakturen und Industriebetriebe. Gerade infrastrukturell gut zu erschließende, direkt an Paris angrenzende Vororte wie Saint-Denis und Ivrysur-Seine erlebten eine beispiellose Veränderung ihrer wirtschaftlichen Ausrichtung und den enormen Zuzug von Franzosen aus der Provinz, die sich ein besseres Auskommen in der Stadt erhofften. Gleichzeitig stärkte die Konstituierung der Republik und die damit einhergehende neue Munizipalgesetzgebung von 1884 die Position der Gemeinden insgesamt und ihrer kommunalen politischen Vertretung im Besonderen. Bildkräftigstes Symbol der Republik, aber auch der politischen Autonomie der Kommunen wurden die neu entstehenden Rathäuser, die den Glanz der Republik repräsentierten und die Identifikation der Bevölkerung mit dem politischen System vorantrieben.

In Ivry-sur-Seine, das durch seinen relativ hohen Industrialisierungsgrad besonders im Bereich der Metallverarbeitung viele Arbeiter anzog, hatten sich vor dem Ersten Weltkrieg kommunalpolitisch vor allem sozialistisch orientierte Parteien durchsetzen können, die die Stadt um die Jahrhundertwende zu einer Hochburg des republikanischen Laizismus machten. Der Erste Weltkrieg stellte insofern eine Zäsur dar, als er auch in Frankreich eine kommunistische Partei hervorbrachte, die den sozialistisch vorbereiteten soziokulturellen Raum in den 1920er und 1930er Jahren politisch besetzte. Als politische Inkarnation des gesellschaftlichen Fortschritts wahrgenommen, mit dem sich solche Katastrophen wie der Erste Weltkrieg hätten abwenden lassen können, gelang es dem PCF, sich in der Pariser banlieue fest zu etablieren. Im Fall Ivry-sur-Seine geschah dies vor allem durch Hilfe im Alltag und die Auflage eines lokalpolitischen Sozialprogramms, das die drängenden Probleme der immigrierten Vorortbevölkerung milderte: Schaffung von kostengünstigem, modernem Wohnraum, Arbeitslosenunterstützung und Kinderbetreuung, insbesondere in den Ferien. Durch diese Lebenshilfen im Alltag entstanden enge Loyalitätsbeziehungen zur nun leninistisch orientierten Parteisektion in Ivry. Diese schuf insbesondere in den Sozialwohnungsbauten eine feste und mobilisierbare Klientel, auf die sie sich bei den politischen Auseinandersetzungen der 1930er Jahre, wie sie meist in Paris als französischer Hauptstadt und Sitz der Deputiertenkammer stattfanden, verlassen konnte.

Zur Sonderrolle Ivrys in der sozialistisch, ab 1935 vor allem kommunistisch regierten Pariser banlieue gehörte auch das politische Personal von orthodoxen Kommunisten, welche bereits 1925 das Rathaus erobern konnten und mit 
Maurice Thorez als Abgeordnetem des Wahlkreises Ivry-Vitry ihren politischen Führer fast täglich fest vor Augen hatten. Unter seiner Herrschaft verdichtete sich nicht nur das lokale kommunistische Milieu, sondern er etablierte auch ideologische Referenzen, welche das Selbstverständnis der Vorstadtbewohner und ihre politische Kultur über Jahrzehnte prägen sollten. Hierzu zählte der Antifaschismusbegriff, welcher eng mit dem Wechsel der Parteistrategie zur Volksfrontoption 1934 auf dem Parteitag in Ivry zusammenhing, die Antikriegshaltung und das damit verbundene internationalistische Engagement und schließlich die patriotische Hinwendung zur französischen Nationalgeschichte, die Ivrys Vorstadtbevölkerung einen klaren Platz in der welthistorischen Entwicklung zuwies.

Der Hitler-Stalin-Pakt und das Verbot des PCF und seiner Vorfeldorganisationen stürzte die Partei, die drei Jahre zuvor bei den Wahlen 1936 und der Volksfrontregierung unter Léon Blum so erfolgreich gewesen war, in eine schwere Krise. Während sich viele Parteimitglieder und kommunistische Stadträte in anderen Vororten, wie vom Pariser Präfekten angeordnet, von der kommunistischen Bewegung distanzierten, war das Bindungsgeflecht in Ivry so stark, dass kein gewählter kommunistischer Stadtrat in Ivry der Partei >abschworく. Als der Präfekt anstelle der gewählten Stadtverordnetenversammlung eine Spezialdelegation einsetzte, zog sich die lokalpolitische Elite in den semilegalen Raum des kommunistischen Milieus zurück.

Die Repressionspolitik der Regierung Daladier führte im Seine-Departement zu überproportional vielen Verhaftungen und Internierungen, denn die Anhänger des PCF konzentrierten sich besonders im roten Gürtel von Paris. Auch Ivrys exponierte Kommunisten waren hiervon betroffen, zumal sie mit der nationalen Führung und den Kadern der mittleren Organisationsebene im Großraum Paris vernetzt und bekannt waren. Mit Beginn der deutschen Besatzung im Sommer 1940 verschärfte sich die schwierige Situation des PCF in Frankreich. Seine Führung unterschätzte den Einfluss der nationalsozialistischen Feindbilder auf das Vorgehen der Besatzungsmacht und drängte seine Kader erst spät in die Illegalität, nachdem die Verhandlungen mit den Deutschen um das Wiedererscheinen der Parteizeitung »L'Humanité« gescheitert waren. In Ivry ging die traditionelle Parteiarbeit mit der Verteilung von Flugblättern und der illegalen »Humanité« trotz der Internierung vieler Bürger und dem Untertauchen der lokalkommunistischen Elite weiter. Die französische kommunistische Partei begann ihren Widerstand gegen die deutsche Besatzung zu systematisieren, als die Wehrmacht im Sommer 1941 die Sowjetunion überfiel. Mit dem Kampf gegen den gemeinsamen Feind wollten Frankreichs Kommunisten die Deutschen im Lande schwächen und so zur Verteidigung der Sowjetunion beitragen. Ivrys suspendierter Bürgermeister Georges Marrane engagierte sich in Südfrankreich beim Aufbau des Front national, sein Beigeordneter Venise Gosnat folgte Marcel Paul als Résistanceverantwortlicher in der Bretagne. Auch in Ivry kam es zu kleineren Widerstandsakten gegen die Wehrmachtssoldaten, allerdings ohne dass sich die Gemeinde 
hierbei quantitativ im Seine-Departement hervorgehoben hätte. Insgesamt hatte die Stadt jedoch eine hohe Zahl von Repressionsopfern zu verbuchen. Von den Verhaftungswellen, Geiselerschießungen, Deportationen und der Haft in deutschen Konzentrationslagern waren mehrere hundert Bürger Ivrys betroffen. Schon während der Kriegshandlungen 1940 waren über fünfundsechzig Soldaten ums Leben gekommen.

Die kommunistische Partei inszenierte das Ende der deutschen Besatzung in Paris im August 1944 als Volksaufstand, in dem die »revolutionäre« Bevölkerung der banlieue eine zentrale, kämpferisch-patriotische Rolle spielte. In Ivry verdeutlichte dies die Rückeroberung des Rathauses unter der Führung von Venise Gosnat. In Paris empfing Georges Marrane als Vize-Präsident des Pariser Befreiungskomitees Charles de Gaulle am 26. August im Hôtel de Ville. Die Herrschaftslegitimation des PCF gewann durch die Rolle der lokalpolitischen Elite und die hohe Zahl der Repressionsopfer in Ivry fortan eine neue Qualität. Ab 1944/1945 entfaltete sich die lokalkommunistische Gedächtnisgemeinschaft. Sie half einerseits, die Zeitspanne von 1939 bis 1944 zu überbrücken, in der die Amtsgeschäfte Ivrys nicht in Händen der kommunistischen Partei, sondern bei einer vom Präfekten eingesetzten, konservativen Spezialdelegation und einem ortsfremden Bürgermeister gelegen hatten. Andererseits sicherte die aufblühende Erinnerungskultur die lokalkommunistische Identität und die damit verbundene Vorherrschaft der Partei langfristig. Die Allianz von lokaler Machtausübung und Herrschaft über die Vorstellung von der Vergangenheit war geschaffen. Das lokalkommunistische Selbstverständnis, das sich nun zusätzlich aus den der Widerstandserfahrung zugeordneten Attributen speiste, wurde in die kulturelle Repräsentanz der Stadt Ivry eingeschrieben und der öffentliche Raum in diesem Sinne umgedeutet. Ein Viertel der Straßen Ivrys erhielten die Namen lokaler und nationaler kommunistischer Repressionsopfer und verorteten ihr Andenken konkret. Gedenksteine an ihren Häusern und Wirkungsstätten markierten das öffentliche Gelände und wiesen Nachbarn, Freunde, Bürger und Passanten permanent auf die früheren Bewohner hin. Kommemorationen und Gedenkzüge nach einem festen Erinnerungskalender forcierten das lokalkommunistische Selbstverständnis. Die persönliche Erinnerung wurde im kommunistischen Milieu aufrechterhalten und über die Gedenkzüge zudem rituell eingebunden. Die lokalkommunistischen Repressionsopfer blieben in der Erinnerung auf den Gedenktafeln nicht anonym, sondern waren mit Namen und Lebensdaten versehen. Ihre Abbildungen fanden sich regelmäßig zu den Gedenkfeiern im »Travailleur«, so dass sie trotz ihrer Zugehörigkeit zum PCF unter Marrane als Individuen wahrnehmbar blieben. Die Ritualisierung und Verankerung der Erinnerung auf Ivrys Stadtgebiet förderten die Identifizierung der Bevölkerung mit ihrer kommunistisch regierten Stadt.

Jürgen Danyel hat einige Ansätze zusammengefasst, mit denen sich der ostdeutsche Antifaschismus und das vermittelte Geschichtsbild der SED cha- 
rakterisieren lassen ${ }^{1}$. Sie weisen neben der starken Ritualisierung der Erinnerung insbesondere in Hinblick auf die Sinngebung der Widerstandstradition Parallelen zu Ivry auf. Danach ergeben sich einige Gemeinsamkeiten, wie eine Überbetonung der Rolle der Partei als treibende Kraft des Widerstandes gegen die nationalsozialistische Herrschaft und die Hervorhebung der politischen Führung - in Ivry von Georges Marrane und Venise Gosnat, die im französischen Fall noch um die Erfindung einer exponierten Rolle von Maurice Thorez in der Résistance ergänzt wurde. In beiden Fällen wurde stark auf die kommunistischen Opfer nationalsozialistischer Gewalt rekurriert, die der Gemeinschaft der Überlebenden eine Sinnrichtung gaben und eine Linearität der Geschichte suggerierten, die es auch in der Zukunft anzustreben gelte. In Ivrys Fall hatte man sich durch die Teilnahme an der Résistance auf die Siegerseite gestellt, sah sich als französischer Teil der Anti-Hitler-Koalition und schien so die »Gesetzmäßigkeiten der Geschichte « bewiesen zu haben. Die Opfer der nationalsozialistischen Gewalt wurden jeweils als die »besten Kräfte des deutschen Volkes« und als die »meilleurs enfants d'Ivry« bezeichnet, deren »Vermächtnis « mit der staatlichen Etablierung der DDR in der deutschen Variante und mit der Fortsetzung des politischen Kampfes in der französischen Variante erfüllt werden sollte. Die pluralistischere Aufstellung der innerstädtischen Gesellschaft bis in die 1950er Jahre wurde dabei nicht mehr reflektiert. Das Andenken konzentrierte sich allein auf die Anhänger und Sympathisanten der kommunistischen Partei und die Durchsetzung ihrer Wiedergutmachungsrechte.

Diese ausgeprägte Erinnerungskultur, an deren wichtigsten Gedenkveranstaltungen in der Nachkriegszeit oft mehrere tausend Menschen teilnahmen, hatte vor allem zwei Konsequenzen: Erstens formte dieser Prozess die politische, soziale und territoriale Identität der Bevölkerung Ivrys nachhaltig und verstärkte die innergemeinschaftlichen Bindungen. Zweitens drängte er die innerörtliche Opposition politisch an den Rand und förderte gleichzeitig die kulturelle und politische Abgrenzung gegenüber dem bürgerlichen und gaullistischen Paris. Die Widerstandsorganisationen und kommunistisch geprägten Veteranenvereine Ivrys orchestrierten diese Entwicklung mit ihren spezifischen Möglichkeiten.

Die Isolation des PCF in der französischen Parteienlandschaft nach 1947, die geographische Distanz zu den sozialistischen Staaten Osteuropas und die schwierige Existenz als kommunistische Enklave im Kalten Krieg unterstützten die Tendenz zu kultureller Abgrenzung und Homogenisierung der Lebensweise in der kommunistischen banlieue. Durch den starken Vergangenheitsbezug unterschied sich Ivry wesentlich von nichtkommunistischen Vororten in der Umgebung. Er unterstrich die Zugehörigkeit zur kommunistischen

1 Jürgen DanYel, Zum Umgang mit der Widerstandstradition und der Schuldfrage in der DDR, in: Ders. (Hg.), Die geteilte Vergangenheit. Zum Umgang mit Nationalsozialismus und Widerstand in beiden deutschen Staaten, Berlin 1995, S.17-104. 
Welt und kennzeichnete sowohl den öffentlichen Raum als auch die lokale Kommunikation. Insbesondere in den 1950er Jahren wurde die Erfahrung von Verfolgung und Widerstand als politisches Argument eingesetzt, etwa gegen die Entsendung des deutschen Generals Hans Speidel in das NATOHauptquartier nach Fontainebleau. Das Prinzip, die Vergangenheit in den Dienst der Gegenwart zu stellen, fand sich aber auch in anderen politischen Konstellationen wieder und stellte die Grundlage für Ivrys Engagement für die Anerkennung der DDR dar.

Obwohl die kulturelle Umdeutung des öffentlichen Raumes und die permanente Erinnerung an die Opfer der Vergangenheit darauf angelegt waren, die politische Identität der Gedächtnisgemeinschaft dauerhaft zu sichern, führten der Generationenwechsel und das Abtreten der Gründergeneration des städtischen Kommunismus in den 1960er Jahren zu einer vorübergehenden Identitätskrise, denn die Frage nach der normativen Tradierung des gemeinschaftseigenen Wissens um die Erfahrung von Widerstand und Verfolgung an zukünftige Generationen stand nun ungelöst im Raum. Nach dem plötzlichen Tod von Maurice Thorez 1964 übernahm Georges Gosnat dessen Wahlkreis Ivry-Vitry. Ein Jahr später folgte Jacques Laloë Georges Marrane im Amt des Bürgermeisters, und auch andere jüngere Männer fanden ihren Platz unter der politischen Elite Ivrys. Kennzeichnend für diese Periode war eine größere Diversität des Gedächtnisses, das nun neuer Formen bedurfte, um die von der Gründergeneration erhaltene kulturelle Grundformation dauerhaft konservieren zu können. Unter Marranes Herrschaft war die lokalkommunistische Erinnerung zwar institutionalisiert, aber vor allem kommunikativ vermittelt worden. Die Erlebnisgeneration war im Alltag und politischen Geschäft stets präsent, die Verfolgungs- und Besatzungszeit als emotional aufwühlendes Ereignis tief in das persönliche Erinnerungsvermögen und die Gedächtnisgemeinschaft eingedrungen. Die neue politische Elite Ivrys war zum großen Teil selbst in diese kulturelle Formation hineingeboren worden und hatte die lokalen Sozialisationsstufen durchlaufen. Zudem blieben die äußeren Rahmenbedingungen - 1939 bis 1944 in Parenthese gestellt - bis in die 1960er Jahre stabil. Die Lebenswelt der milieugebundenen Arbeiterschaft wies insgesamt starke Kontinuitäten auf.

In der Umbruchsphase der 1960er Jahre wurde bewusst nach neuen Ausdrucksformen des lokalkommunistischen Gedächtnisses gesucht. Dieses Reflexiv-Werden erweiterte den Modus des kulturellen Gedächtnisses, das zwar seine Kommunikationsfunktion beibehielt und in den Kommemorationsveranstaltungen gepflegt wurde, nun aber weitere Stützen erhielt, um die kulturelle Kohärenz zu wahren und seine Inhalte nicht aus dem Erinnerungsrahmen fallen zu lassen. Dies zeigte sich in der Erweiterung und Anpassung des innerstädtischen Erinnerungskalenders und der Kanonisierung historischer Überlieferung, welche die relevanten Pfeiler des stadteigenen Selbstverständnisses ausmachten. Hatten bis dahin die städtischen Repressionsopfer aus dem kommunistischen Milieu im Mittelpunkt des Gedenkens gestanden und 
die Gedenktage geprägt, fanden nun auch außerstädtische Bezüge Eingang in die Gedächtnisgemeinschaft. So wurde der 8. Mai als gemeineuropäisches Datum des Sieges über das nationalsozialistische Deutschland in Ivrys Gedächtnis immer wichtiger, zumal sich hier Anknüpfungspunkte für eine gemeinsame Erinnerung mit der Sowjetunion boten. Das Andenken an die jüdischen Opfer des nationalsozialistischen Rassenwahns, wovon auch einige aschkenasische Familien aus Ivry betroffen waren, fand zögerlich Aufnahme in die Gedenktage und wurde von der kommunistischen Identität überlappt. In den 1970er und frühen 1980er Jahren wurden in erster Linie jüdische Widerstandsakte rezipiert. Der Schwerpunkt lag auf dem kämpferisch-aufständischen Element, aus dem sich das eigene lokalkommunistische Selbstverständnis speiste. Dieses hatte sich gleichzeitig in kanonisierender Art auf einige Topoi zugespitzt. Ivry wurde zur Märtyrerstadt stilisiert, die sich vor diesem Legitimationshintergrund in die Belange der Welt einmischte und den eigenen Standpunkt im welthistorischen Koordinatensystem durch eine ausgeprägte Symbolpolitik vertrat. 1936 hatte Ivrys Stadtrat bereits die Ehrenbürgerschaft an den inhaftierten deutschen Kommunistenführer Ernst Thälmann verliehen, 1971 wurde sie an Franz Dahlem und seine Frau Catherine vergeben, Ende der 1980er Jahre wurde der südafrikanische Freiheitskämpfer Nelson Mandela trotz seiner Inhaftierung zum Ehrenbürger Ivrys ernannt.

Während diese Identitätskomponenten im Rahmen des modifizierten Erinnerungskalenders zumeist kommunikativ vermittelt wurden, drückte sich die Erweiterung des kulturellen Gedächtnisses in den 1960er Jahren durch zusätzliche Gedächtnisstützen und neue Institutionen im öffentlichen Raum aus. Hierzu gehörte die Initiative zum Bau eines Museums für den nationalen Widerstand, dessen Errichtung zu einer erheblichen lokalen, nationalen und transnationalen Mobilisierung der Bevölkerung und Institutionen auch jenseits der französischen Grenzen führte. In der Tradition eines Aufrufs von Maurice Thorez an die Bevölkerung Ivrys inszeniert, das Andenken an die Résistance zu wahren und damit seinen letzten Willen zu erfüllen, bildete die Museums- und Tradierungsfrage eine wesentliche Beschäftigung der neuen Mannschaft im Rathaus. Nachdem sich die Realisierung des Museums in Ivry über Jahre hingezogen hatte und man schließlich seine Errichtung in einem Nachbarort hinnehmen musste, bot die Gründung des Stadtarchivs im Keller des Rathauses Anfang der 1980er Jahre der Stadt Ivry eine zusätzliche Möglichkeit, lokalhistorische Erfahrung zu konservieren und weiterzugeben.

Die allgemeine Zunahme internationaler Kontakte in den 1950er und 1960er Jahren spiegelte sich auch in den kommunistischen Vorstädten von Paris wider und dokumentierte in Ivrys Fall die transnationale Erweiterung der Gedächtnisthematik. Die Verankerung Ivrys im Koordinatensystem der kommunistischen Weltbewegung und der französischen Nationalgeschichte gehörte spätestens seit den 1930er Jahren zu einer der wichtigsten, der lokalen Gemeinschaft vermittelten Sinngebungen. Der Wechsel von einem stark parlamentarisch geprägten zu einem präsidentiellen Regierungssystem 1958 
und der Beginn einer relativ autonomen französischen Außenpolitik unter Staatspräsident de Gaulle führten im Spannungsfeld der Ost-West-Beziehungen zu veränderten Rahmenbedingungen. Nun konnten sich neue Formen lokalkommunistischer Traditionsbildung entwickeln, die über die abgesteckten Grenzen kommunaler Erinnerung hinausgingen. Diese Öffnung nach außen vollzog sich allerdings nicht gegenüber der französischen Mehrheitsgesellschaft, von der sich das kommunistische Milieu weiterhin abgrenzte, sondern entlang der städtischen Herrschaftsstrukturen mit ihren Verbindungen in die französische Parteispitze und ins sozialistische Ausland.

Zwei Pfade wurden zur Intensivierung der Beziehungen mit dem Ausland beschritten. Zum einen ging Ivrys Sektion der französischen Deportiertenvereinigung FNDIRP 1961 eine Partnerschaft mit dem NS-Verfolgtenverband VVN Essen in der Bundesrepublik Deutschland ein. Zum anderen verdichteten sich die Kontakte mit der DDR. Ideologisch über den traditionellen Internationalismus der Arbeiterbewegung, aber auch durch die kommunistische Biographie des KPD/SED-Funktionärs Franz Dahlem mit dem zweiten deutschen Staat verbunden, entstand 1963 eine Städtepartnerschaft zwischen Ivry und Brandenburg an der Havel. Das Andenken an die Opfer des Nationalsozialismus nahm bei der Annäherung zwischen beiden Städten wesentlichen Raum ein und wurde in Erinnnerungsritualen gepflegt. Zudem förderte die verblassende Anziehungskraft der Sowjetunion sowie eine ähnliche, homogenisierend wirkende Sozial- und Erziehungspolitik die Identifizierung Ivrys mit der DDR. Französische Delegationen nahmen regelmäßig am OdF-Tag in Brandenburg oder an anderen Gedenkveranstaltungen in der Umgebung teil. Besuche von Erinnerungsorten wie der ehemaligen Konzentrationslager Sachsenhausen, Ravensbrück und Buchenwald in der DDR gehörten zum obligaten Besuchsprogramm und bestätigten die Besucher in ihrer eigenen Identität als Opfer nationalsozialistischer Gewalt über die Grenzen des Ost-WestKonflikts hinaus. Wenngleich die antifaschistische und friedensorientierte Selbstdarstellung der DDR auf starke Resonanz im kommunistischen Milieu stieß und die SED hier einen wichtigen Verbündeten für die Verbreitung eines positiven DDR-Bildes gefunden hatte, zeigten sich nach der integrierend wirkenden Anerkennungsphase unterschiedliche Vorstellungen bei den Opferorganisationen in Ost und West, wie intensiv die Erinnerung tagespolitisch aufgeladen werden sollte. Während der Antifaschismus der DDR in den 1980er Jahren zum Ritual erstarrt und in seiner permanenten Repetition Ausdruck ihrer »kulturellen Erschöpfung « (Jan-Holger Kirsch) geworden war, erlebte sein französisches Äquivalent in Ivry Modifikationen und passte sich den historischen Themen und Umständen an. So reflektierten die städtischen Erinnerungsrituale etwa die Öffnung des PCF zur sozialistischen Partei, mit welcher 1972 ein gemeinsames Regierungsprogramm ausgearbeitet wurde. Allerdings blieb die Prädominanz der kanonisierten kommunistischen Erinnerung im munizipalen Zusammenhang stets erhalten. Ihr Einfluss auf die Bevölkerung und die Entwicklung ihres historischen Bewusstseins blieb über 
Jahrzehnte stabil, auch wenn die Zahl der Kommemorationsteilnehmer nicht mehr in die Tausende ging wie in der unmittelbaren Nachkriegszeit.

In der stark ausgeformten, anpassungsfähigen Erinnerungskultur lag schließlich auch ein Erklärungsmoment für die mangelnde Wahrnehmung der Verfallstendenzen in der Sowjetunion und in der DDR, von deren Zusammenbruch Ivrys Kommunisten völlig überrascht wurden. Die ständige Präsenz der Vergangenheit, ihre normierende Wirkung und das verfestigte, zwar modulierbare, aber dennoch polarisierende Weltbild der lokalkommunistischen Erinnerungsgemeinschaft immunisierte gegen äußere Einflüsse und reflektierte zugleich die mangelnde Sensibilität gegenüber der Entwicklung in Osteuropa. Dass die Signale des Zusammenbruchs zudem aus Staaten mit utopischem Vorbildcharakter für die eigene Lebenswelt kamen, machte ihre Erkenntnis umso schwieriger. Denn sie hätte die Sinnhaftigkeit der kommunistischen Identität und ihren Weg in die Zukunft nachhaltig in Frage gestellt.

In der Pariser banlieue blieb das Gedächtnis im öffentlichen Raum lebendig und die mannigfaltig orchestrierte Umdeutung Ivrys zur Märtyrerstadt war ein Grund für den dauerhaften Erfolg der kommunistischen Partei. Denn über das Instrument der Erinnerungskultur war es ihr gelungen, die politische Identität und das Selbstbild der Bevölkerung an die Stadt Ivry zu binden, so dass auch die umgekehrte Gleichung Ivry gleich kommunistisches Terrain eine feste Bezugsgröße für die nachfolgenden Generationen und potentiellen Wähler wurde. Auch nach dem Generationenwechsel konnte die kommunistische Partei in Ivry so die kulturelle Kohärenz wahren. Im Unterschied zur erstarrten Erinnerungskultur der DDR erwies sich Ivrys kollektives Gedächtnis nach 1964/65 als sehr anpassungsfähig an die jeweiligen Zeitumstände. Zwar blieb die kulturelle Grundformation erhalten und prägte die lokalkommunistische Perspektive auf die Vergangenheit weiterhin, diese wurde nun jedoch den aktuellen politischen und gesellschaftlichen Rahmenbedingungen stärker angepasst, was umgekehrt das Identifikationsangebot der kommunistischen municipalité erweiterte und als kulturelle Praxis lebendig blieb.

Der allgegenwärtige und jederzeit abrufbare Vergangenheitsbezug Ivrys könnte schließlich eine Erklärung dafür bieten, dass in diesem lokalen Rahmen der Unterschied von Demokratie und Diktatur gar nicht wahrgenommen und reflektiert wurde. Aus der Munizipalgesetzgebung von 1884 und der besonderen historischen Situation der ersten Nachkriegszeit hervorgegangen, konnte sich der städtische Kommunismus auf dem Boden der Republik entfalten, er schien sogar in der angenommenen historischen Gesetzmäßigkeit eine logische Konsequenz zu sein. Die ausgeprägte Erinnerungskultur nach 1944/45 stellte die historische Kontinuität zur Dritten Republik her und hielt die traditionellen Feindbilder aufrecht, so dass die Besatzungszeit als Parenthese, nicht als historischer Bruch erschien. Diese spezielle Konstellation in der zweiten Nachkriegszeit hatte weiterhin die Republik als systemischen Bezug. Die dem städtischen Kommunismus innewohnenden diktatorischen Züge wurden nicht als undemokratisch problematisiert und ließen auch die Dikta- 
turen in Osteuropa nicht als solche erkennen. Vielmehr bestätigten die Parallelisierung Ivrys mit der DDR und die Identifizierung mit ihrer lokalen Verwaltung die gefühlte Zugehörigkeit Ivrys zur kommunistischen Welt, deren Existenzbedingungen mit den eigenen gleichgesetzt wurden. Im Gegensatz zu den sozialistischen Staaten war Ivry jedoch stärker mit seiner Außenwelt vernetzt, konnte sich dem gesellschaftlichen Wandel in den 1970er und 1980er Jahren nicht verschließen und zeigte eine größere Anpassungsleistung, die sich nicht nur in der kulturellen Repräsentanz, sondern auch in der gezielten Förderung der Ansiedlung von Industrie- und Dienstleistungsunternehmen ausdrückte. Dass trotz des Zusammenbruchs der kommunistischen Welt in Osteuropa und der in den 1990er Jahren auftretenden massiven Kritik an der französischen Linken ${ }^{2}$ in Ivry weiterhin ein kommunistischer Stadtrat mit absoluter Mehrheit die Geschicke der Vorstadt lenkt, ist sicherlich auch auf die historische Legimitation seiner lokalpolitischen Herrschaft und die fortdauernde Bindung der Wählerklientel zurückzuführen.

2 Courtors, Du passé; Furet, Le passé. 